\title{
Theoretical Estimation of Absorption Coefficients of Various Polymers at $13 \mathrm{~nm}$
}

\author{
Nobuyuki N. Matsuzawa*, Hiroaki Oizumi*, Shigeyasu Mori"**, Shigeo Irie"***, Ei Yano****, Shinji \\ Okazaki** and A kihiko Ishitani"******
}

Atsugi Research Center, Association of Super-Advanced Electronics Technologies, clo NTT Atsugi $R$ \& D Center,

3-1 Morinosato-Wakamiya, Atsugi 243-0198, Japan

Permanent Address: * Frontier Science Laboratories, SONY Corporation, "* Central Research Laboratory, Hitachi Ltd., ${ }^{* * *}$ Central Research Laboratory, Sharp Corporation, ${ }^{* * *}$ ULSI Process Technology Center, Matsushita Electronics Corporation, ${ }^{* * * * *}$ Fujitsu Laboratories Ltd. ${ }^{* * * * *}$ NEC Corporation

\begin{abstract}
By using the mass absorption coefficients of Henke at $13 \mathrm{~nm}$ and the density obtained from the graph-theoretical treatment derived by Bicerano et al., we have calculated the linear absorption coefficients at $13 \mathrm{~nm}$ for about 150 polymers. The computational time required for a prediction is at most a few minutes, including the time to construct the input deck. The mean error of the predicted values is estimated to be $2.2 \%$. Thus, a method of quickly surveying polymers to find those with a low absorption at $13 \mathrm{~nm}$ has been established.
\end{abstract}

Keywords: EUV Lithography, linear absorption coefficient, photoresist

\section{Introduction}

Extreme ultraviolet (EUV) lithography at a wavelength of $13 \mathrm{~nm}$ is a promising candidate for the fabrication of patterns with feature sizes of $100 \mathrm{~nm}$ and below. [1-3] One of the most important parameters in the design of photoresist materials for this type of lithography is the absorption coefficient of a polymer because, if the polymer is not transparent enough, the resist pattern deteriorates. Thus, as the starting point of our theoretical study on EUV resist polymers, we have constructed a tool that provides a reasonably accurate prediction of the absorption coefficient in the EUV region.

Previous studies [4-6] have shown that the absorption coefficient of a polymer film in the EUV region can be calculated quite simply from the density of the polymer and the sum of the atomic absorption cross sections of the atoms contained in the polymer. For example, the linear absorption coefficient of PMMA (polymethylmethacrylate) estimated in this way using the measured density of $1.275 \mathrm{~g} / \mathrm{cm}^{3}$ has been reported to be $5.3 \mu^{-1}$. This value is in reasonable agreement with experimentally obtained values $\left(5-6 \mu^{-1}\right)$, that is, the estimated value falls within the range of experimental values. [5] A similar good agreement between the experimental and the estimated value has been reported for the Hoechst AZ PN1 14 resist, using a measured density of $1.2 \mathrm{~g} / \mathrm{cm}^{3}$. For this resist, the experimental value at $14 \mathrm{~nm}$ has been reported to be $4.4 \pm 1 \mu^{-1}$, whereas the estimated value is $4.8 \mu^{-1}$. [5] Thus, for the prediction of the absorption coefficient, density is the only quantity that remains to be calculated theoretically.

\section{Calculations}

This study involved the application of the graph theoretical treatment of molecules derived by Bicerano et al. to the calculation of the density of a polymer. [7-9] This method employs an empirical correlation between the properties and connectivity index of polymers. The Synthia program module in the POLYMER program system [10] was used for the calculations; it provides a mean error of $2.2 \%$ for density 
predictions. [7] The linear absorption coefficient at $13 \mathrm{~nm}$ was then calculated using the following equation.

$$
\Sigma \mu_{1}=\Sigma \mu_{\mathrm{a}} \times\left(\mathrm{N}_{\mathrm{o}} \rho / \mathrm{A}\right)
$$

where $\mu_{1}$ is the linear absorption coefficient, $\mu_{\mathrm{a}}$ is the mass absorption coefficient, $N_{o}$ is Avogadro's number, $A$ is the atomic weight and $\rho$ is the mass density of the absorber. Note that the sum $\Sigma$ in eq (1) was taken over the atoms contained in the polymer. Note also that the values of $\mu_{\mathrm{a}}$ used in our study are those obtained by Henke et al. [11] All of the calculations was done on an SGI/COMTEC 4D OCTANE R10000/250 $\mathrm{MHz}$ SSE workstation.

\section{Results and Discussion}

\subsection{Time Required for the Prediction}

In order to benchmark the time required for the prediction, we chose PMMA homopolymer and also a copolymer of tricyclodecanylacrylate, ethoxyethylmethacrylate and methacrylic acid (Figure 1). Table I shows the calculated linear absorption coefficient obtained from the theoretical density and the time required for the calculation, which is not the CPU time, but rather the total time actually required, including the time for making the input deck. Figure 2 shows calculated spectra of PMMA and the copolymer in the EUV region.

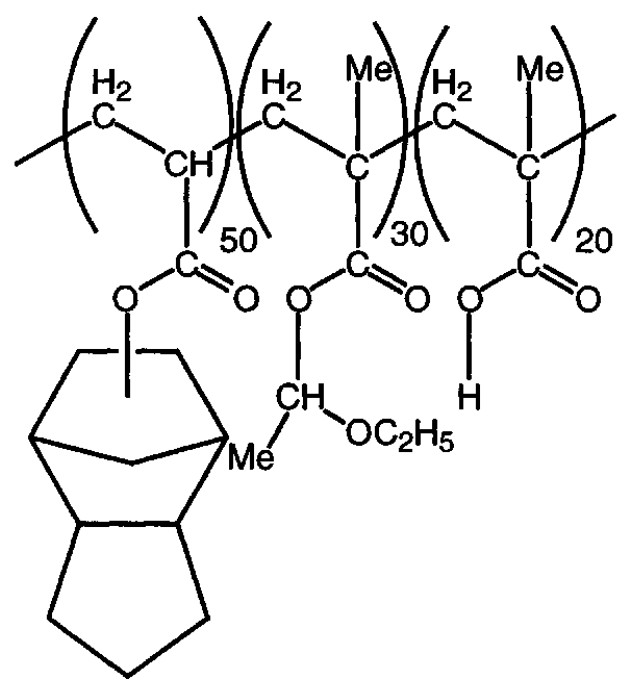

Figure 1. Copolymer used to calculate benchmark

Table I shows that the calculations for the homopolymer only take a couple of minutes. The predicted value is $4.80 \mu^{-1}$. This is in good agreement with the experimental value of $5-6 \mu^{-1}$,
[5] but it is slightly smaller because the predicted density for PMMA $\left(1.159 \mathrm{~g} / \mathrm{cm}^{3}\right)$ is smaller than the experimental value $\left(1.275 \mathrm{~g} / \mathrm{cm}^{3}\right)$.

Table I. Calculated linear absorption coefficient and the time required for the prediction.

Polymer Absorption coefficient Time required

$\left(\mu^{-1}\right)$

(min:sec)

\begin{tabular}{llr}
\hline PMMA & 4.80 & $1: 45$ \\
Copolymer & 4.11 & $13: 30$ \\
\hline
\end{tabular}

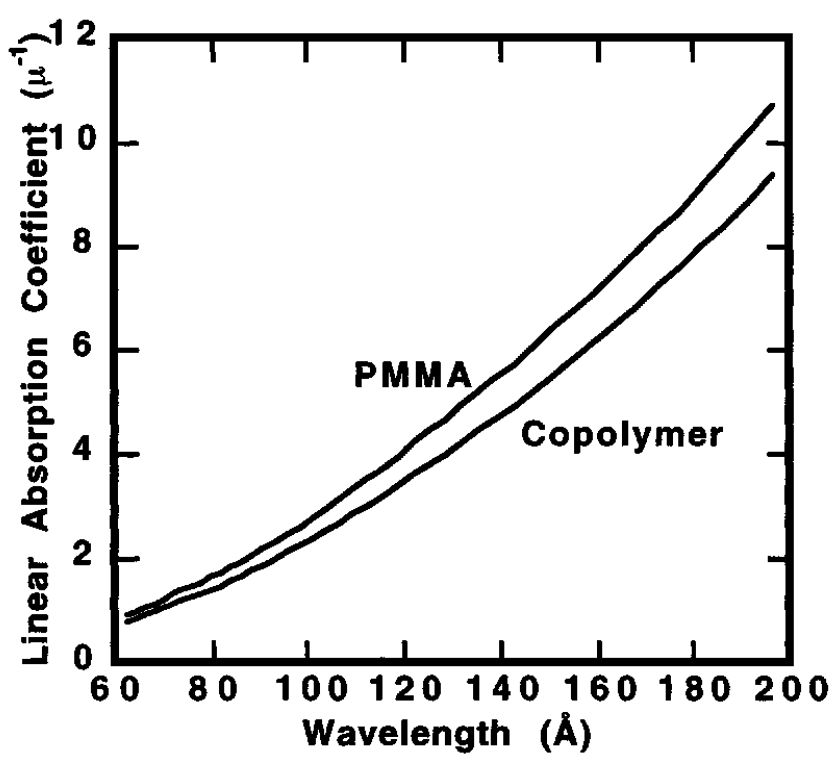

Figure 2. Calculated EUV absorption spectra of PMMA and the copolymer.

Even for the copolymer, which has a significantly more complicated structure than PMMA, the time actually required for the prediction is only about 10 minutes. In this case, the predicted density was $1.134 \mathrm{~g} / \mathrm{cm}^{3}$, and the linear absorption coefficient is smaller than that of PMMA.

These results show that the method of theoretically predicting linear absorption coefficients presented here is a very useful and practical tool for surveying polymers to find those with a low absorption at $13 \mathrm{~nm}$, and is much, much quicker than doing experiments.

\subsection{Prediction of the linear absorption} coefficients of various polymers

We than proceeded to predict the linear absorption coefficients of the various polymers listed in Table II. They are mainly hydrocarbon 
polymers containing oxygen atoms, such as PMMA, but they also include silicon containing polymers and fluorinated polymers.

Table II. List of calculated polymers

polyacenaphthylene, polyacetal, polyacrylamide, polyacrylic acid, polyacrylonitrile, polybenzylacrylate, polybenzylmethacrylate, polybutadiene, poly(1-butene), poly(2-butoxyethyl acrylate), poly(n-butyl acrylate), poly(t-butyl acrylate), poly(t-butylaminoethylmethacrylate), poly(nbutyl methacrylate), poly(sec-butyl methacrylate), poly(t-butyl methacrylate), poly(1,4-butylene adipate), poly(1,4-butylene terephthalate), poly (t-butyl styrene), polycaprolactone, polycarbonate, poly(cylcohexyl acrylate), poly (cylcohexyl methacrylate), poly(n-decyl acrylate), poly(n-decyl methacrylate), poly(di-t-butyl vinylpiridine), poly(2,6-dimethyl-1,4-phenylene oxide), succinate), poly (2,2-dimethyl-1,3-propylene poly(dimethylaminoethyl methacrylate), poly(2,3-dihydrofuran), polydiethoxysiloxane, polydiethylsiloxane, polydimethylsiloxane, polydimethylsilane, poly $\left(4,4^{\prime}\right.$ dipropoxy-2,2'-diphenylpropane fumarate), poly(2-(2-ethoxyethoxy)-ethyl acrylate), poly (ethyl acrylate), poly(ethyl methacrylate), polyethylene, poly(ethylene adipate), poly (ethylene azelate), poly(ethylene glycol), poly (ethylene succinate), poly(ethylene terephthalate), poly(2-ethylhexyl acrylate), poly(2-ethylhexyl methacrylate), poly(ethyl methacrylate), poly(hexadecyl methacrylate), poly(hexafluoropropylene oxide), poly(hexamethylene sabacate), poly(n-hexyl acrylate), poly(n-hexyl methacrylate), poly(p-hydroxy benzoate), poly (hydroxybutyric acid), poly(4-hydroxybutylacrylate), poly(2-hydroxybutylacrylate), poly(2hydroxyethylmethacrylate), poly(2-hydroxypropylacrylate), poly(2-hydroxypropylmethacrylate), poly(4-hydroxystyrene), polyimide, poly(isobornyl-acrylate), poly(isobornylmethacrylate), poly (isobutyl acrylate), polyisobutylene, poly (isobutyl methacrylate), polyisoprene, poly(isopropyl acrylate), poly (isopropyl methacrylate), poly(lauryl acrylate), poly(lauryl methacrylate), polymethacrylamide, poly(methacryl acid), polymethacrylonitrile, poly (2-methoxyethy acrylate), poly(4-methoxystyrene), poly(methyl acrylate), poly(methyl methacrylate), poly(methylhexadexylsiloxane), poly(methyl-hexylsiloxane), poly(methylhydro- silane), poly(methylhydrosiloxane), poly (methyloctadexylsiloxane), poly(methyloctylsiloxane), poly(methylphenylsiloxane), poly(4methyl-1-pentene), poly ( $\alpha$-methylstyrene), poly (4-methylstyrene), poly(methyltetradecylsiloxane), poly(neopentylmethacrylate), poly (neopentyl glycol sebacate), poly(octadecyl acrylate), poly(octadecyl methacrylate), poly (octyl_acrylate), poly(octyl_methacrylate), poly (2-phenoxyethyl acrylate), poly(2-phenoxyethyl methacrylate), poly(2-phenyl methacrylate), poly (phenylethyl methacrylate), poly(propyl methacrylate), poly(propyl acrylate), poly (propylene), poly(propylene glycol), polystyrene, polytetrafluoroethylene, polytetrahydrofuran, poly(tetrahydrofurfuryl methacrylate), poly (tetrahydrofurfuryl acrylate), poly(tridecyl methacrylate), poly(trimethylene adipate), poly (trimethylene glutarate), poly(trimethylene succinate), poly(vinyl acetate), poly(vinyl alcohol), poly(vinyl biphenyl), poly(vinyl carbazole), poly(vinyl cinnamate), poly(vinyl ethylether), poly(vinyl fluoride), poly(vinylidene fluoride), poly(vinyl laurate), poly(vinyl methylether), poly(vinyl methylketone), poly(vinyl naphthalene), poly(vinyl pivalate), poly(vinyl propionate), poly(2-vinyl piridine), poly(vinyl stearate), Nylon-6, Nylon-6/6, Nylon$6 / 9$, Nylon-6/10, Nylon-6/12, Nylon-11, Nylon12

A plot of the calculated linear absorption coefficient versus the calculated density is shown in Figure 3. The trend is generally linear, with the linear absorption coefficient becoming larger as the density increases. This is indicated in the figure by the "trend line". This trend is explained by the Eq. (1), in which the contribution of a particular kind of atom to the linear absorption coefficient is directly proportional to the density of the atoms. It is clear that low-density polymers are better for obtaining a low absorption at $13 \mathrm{~nm}$.

Figure 3 also shows that, for the fluorinated polymer polyvinylfluoride, the calculated coefficient is larger than that expected from the trend line. This is because the atomic absorption of fluorine at $13 \mathrm{~nm}$ is larger than that of the other atoms like $\mathrm{C}, \mathrm{H}, \mathrm{O}, \mathrm{N}$ and $\mathrm{Si},[11]$ and the density does not compensate the larger atomic absorption of this polymer. The same kinds of results were also obtained for other fluorinated polymers, such as poly(hexafluoropropylene 


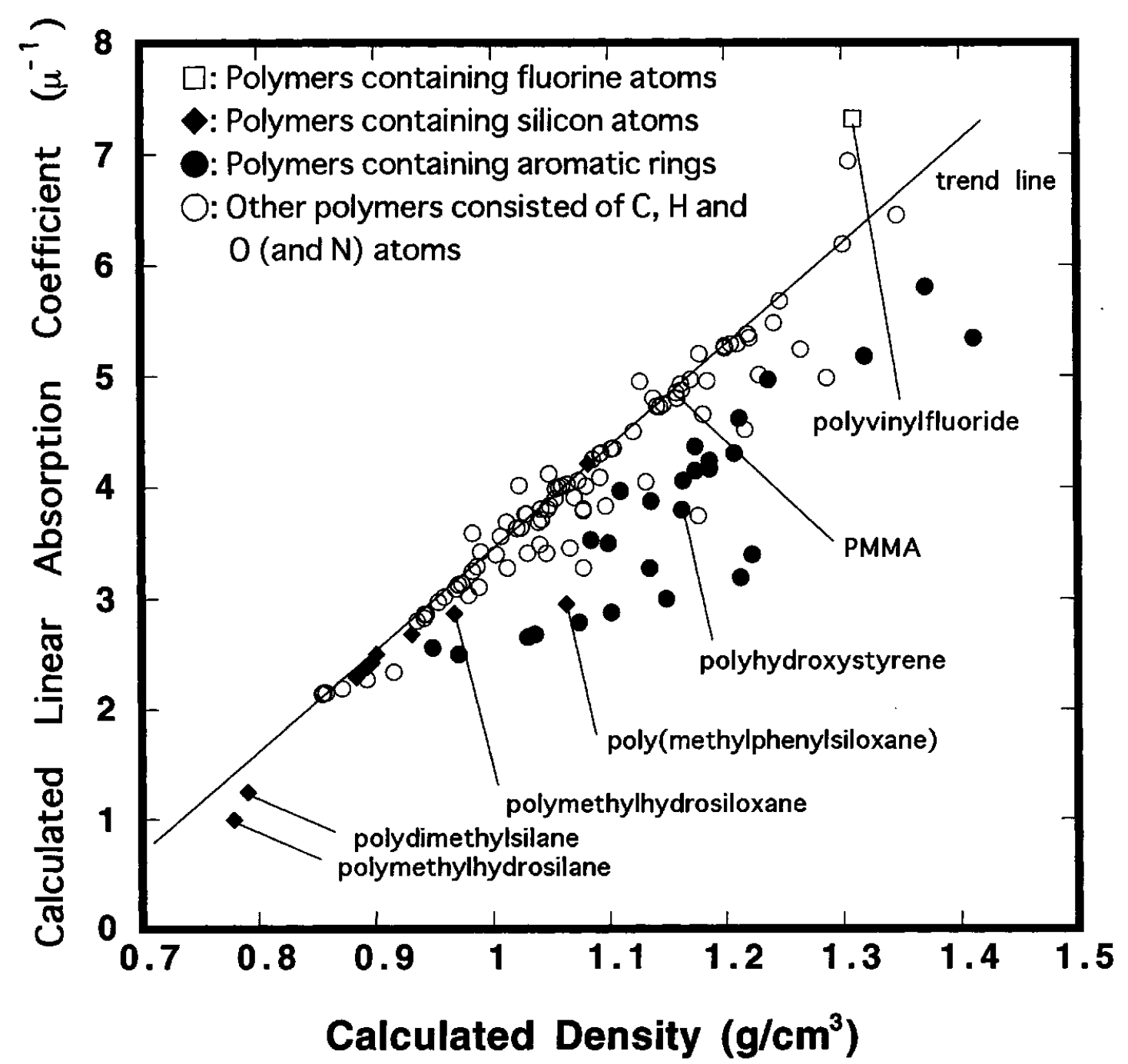

Figure 3. Calculated linear absorption coefficient versus calculated density.

oxide) (Abs: $16.1 \mu^{-1}, \rho=2.02 \mathrm{~g} / \mathrm{cm}^{3}$ ), polytetrafluoroethylene (Abs: $16.4 \mu^{-1}, \rho=2.05$ $\mathrm{g} / \mathrm{cm}^{3}$ ), and poly(vinylidene fluoride) (Abs: 11.0 $\left.\mu^{-1}, \rho=1.61 \mathrm{~g} / \mathrm{cm}^{3}\right)$, although the numbers of these polymers are not shown in the figure (Abs: linear absorption coefficient).

For some hydrocarbon polymers (containing oxygen atoms), the calculated linear absorption coefficient is lower than that expected from the trend line. Most of the polymers below the trend line contain aromatic rings, such as benzene and naphthalene rings. It is known that oxygen atoms exhibit a larger atomic absorption at $13 \mathrm{~nm}$ than $\mathrm{C}$ or $\mathrm{H}$ atoms. [11] This means that, as the oxygen content of a polymer becomes higher, the linear absorption coefficient should also become higher, provided that there is no significant change in density. That is, a polymer with aromatic rings has a lower oxygen content than one without, thereby resulting in a lower absorption.

For silicon containing polymers, silane polymers of polymethylhydrosilane and polydimethylsilane show the lowest values of 1.00 $\mu^{-1}$ and $1.25 \mu^{-1}$, respectively, as compared to those for the other all. The data for these two polymers in Figure 3 is located below the trend line, and this is because the atomic absorption of $\mathrm{Si}$ atom is smaller than those of $\mathrm{C}, \mathrm{H}$ and $\mathrm{O}$ atoms. [11]

Calculations were also done on siloxane polymers. The data for these polymers is generally located close to the trend line. This is because the low atomic absorption of silicon atoms is compensated for to some extent by the large atomic absorption of the oxygen atoms present in siloxane polymers. The only siloxane polymer below the trend line is 
poly(methylphenylsiloxane); and the reason is that it contains an aromatic ring.

\section{Conclusion}

By using the mass absorption coefficients of Henke et al. at $13 \mathrm{~nm}$ and the density obtained from the graph-theoretical treatment derived by Bicerano et al., we have calculated the linear absorption coefficients of about 150 polymers at $13 \mathrm{~nm}$. The computational time required for a prediction is at most a few minutes, including the time needed to construct the input deck. The mean error of the predicted values is estimated to be $2.2 \%$. This demonstrates that a method of quickly surveying polymers to find those with a low absorption at $13 \mathrm{~nm}$ has been established.

Our calculations show that polymers with a low density make better EUV photoresists in terms of photo-absorption. They also show that fluorinated polymers are not so suitable because of their large linear absorption coefficient. Furthermore, it was found that one way to reduce the absorption at $13 \mathrm{~nm}$ is to introduce an aromatic ring into a polymer. Silane polymers were found to exhibit the lowest linear absorption coefficient, so from the standpoint of absorption, these polymers make the best EUV photoresists. Siloxane polymers also have a lower absorption than the others; but this is mainly due to their low density. The advantage arising from the low atomic absorption of silicon atom does not exist when siloxane polymers are used.

\section{A cknowledgment}

This work was performed under the management of the Association of SuperAdvanced Electronics Technologies (ASET) as part of the Ministry of International Trade and Industry (MITI) Program of Super-Advanced Electronics Technologies supported by the New
Energy and Industrial Technology Development Organization (NEDO). The authers are grateful to Mr. H. Setoya for valuable discussions

\section{References}

1. T. Watanabe and H. Kinoshita, FED J., 8 (1997) 51.

2. A. M. Hawryluck, N. M. Ceglio and D. A. Markle, Microlithogr. World, Summer (1997) 17.

3. A. M. Hawryluck, N. M. Ceglio, and D. A. Markle, Solid State Technol., Aug (1997) 75, and 151.

4. W. M. Mansfield, J. E. Bjorkholm, A. A. MacDowell, R. R. Freeman, L. H. Szeto, G. N. Taylor, D. M. Tennant, W. K. Waskiewicz, D. L. Windt, D. L. White, O. R. Wood II, R. M. D'Souza and A. R. Neureuther, OSA Proc. Soft-X-Ray Projection Lithogr., 12 (1991) 129.

5. G. D. Kubiak, E. M. Kneedler, R. Q. Hwang, M. T. Schulberg, K. W. Berger, J. E. Bjorkholm and W. M. Mansfield, J. Vac. Sci. Technol. B, 10 (1992) 2593.

6. G. M. Wells, J. W. Taylor, F. Cerrina, D. Pearson and J. MacKay, J. Vac. Sci. Technol. B, 10 (1992) 3252.

7. J. Bicerano, Predictions of the Properties of Polymers from their Structures, Marcel Dekker: New York, 1993.

8. L. B. Kier and L. H. Hall, Molecular Connectivity in Chemistry and Drug Research, Academic Press: New York 1976.

9. L. B. Kier and L. H. Hall, Molecular Connectivity in Structure-Activity Analysis, JohnWiley \& Sons: New York (1986).

10. This program is available from MSI Inc., San Diego, CA.

11. B. L. Henke, E. M. Gullikson and J. C. Davis, Atomic Data and Nuclear Data Tables, 54 (1993) 181. 